Rev. Adm. Saúde (On-line), São Paulo, v. 21, n. 82: e283, jan. - mar. 2021, Epub 03 abr. 2021 http://dx.doi.org/10.23973/ras.82.283

ARTIGO ORIGINAL

\title{
Informação como apoio para tomada de decisão de gestores públicos de saúde
}

Information as support for decision making by public health managers

Fábio Moschen Antunes', Josué Souza Gleriano², Bruna Moreno Dias ${ }^{3}$, André Almeida de Moura ${ }^{4}$, Liz Vanessa Lupi Gasparini ${ }^{5}$

\author{
1. IDS Softwares \\ 2. Universidade do Estado de Mato Grosso \\ 3. Escola de Enfermagem de Ribeirão Preto da Universidade de São Paulo \\ 4. Escola de Enfermagem de Ribeirão Preto da Universidade de São Paulo \\ 5. Universidade do Estado de Mato Grosso
}

\section{RESUMO}

Para exercer a gestão em saúde os gestores precisam alinhar a tomada de decisão às habilidades da administração. O uso dos Sistemas de Informação em Saúde (SIS) é imprescindível nesse processo. Esse estudo teve por objetivo analisar, a partir do suporte de assessoria de informação, as demandas de gestores públicos de saúde para a tomada de decisão. Trata-se de uma pesquisa documental descritiva apoiada no método de estudo de caso múltiplo, por meio da base de dados de uma empresa de desenvolvimento de software e assessoria que atende secretarias municipais de saúde no território nacional. Os dados foram extraídos do sistema de informações da empresa considerando os chamados realizados pelos gestores de saúde. Os dados foram analisados a partir da análise temática com utilização do software Nvivo. Em relação às demandas dos gestores, predominaram solicitações de informações relacionadas às Unidades da Estratégia de Saúde da Família 
(ESF) e ao Pronto Atendimento (UPA), especialmente aquelas relacionadas à assistência ao usuário. Dentre as solicitações, destacaram-se aquelas pertinentes à produção de serviços e monitoramento do cuidado, oriundos das coordenações de atenção à saúde e da vigilância em saúde. A empresa emitiu relatórios como forma de resposta às demandas. Ressalta-se que a análise das demandas proporciona oportunidade de compreender o uso dos dados para a tomada de decisão e possibilidade de refletir as dificuldades técnicas dos gestores no uso dos SIS. Essa análise contribuiu para a empresa de assessoria identificar a necessidade de desenvolvimento de um plano de capacitação para os gestores.

Palavras-chave: Gestão em Saúde; Sistemas de Informação em Saúde; Aplicações da Informática Médica; Administração Municipal; Sistemas Locais de Saúde.

\begin{abstract}
To exercise health management, managers need to align decision making with management skills. The use of Health Information Systems (HIS) is essential in this process. This study aimed to analyze, based on the support of information advisory services, the demands of public health managers for decision making. This is a descriptive documentary research supported by the multiple case study method, through the database of a software development and advisory company that serves municipal health secretariats in Brazil. The data were extracted from the company's information system considering the calls made by health managers. The data were analyzed using thematic analysis with the Nvivo software. In relation to the managers' demands, there was a predominance of requests for information related to the Family Health Strategy Units (ESF) and to the Emergency Room (UPA), especially those related to user assistance. Among the requests, the most important were those related to the production of services and monitoring of care, from the health care and health surveillance coordinations. The company issued reports as a form of response to the demands. It is noteworthy that the analysis of the demands provides an opportunity to understand the use of data for decision making and the possibility to reflect the technical difficulties of managers in using the HIS. This analysis helped the consulting firm to identify the need to develop a training plan for managers.
\end{abstract}

Keywords: Health Management; Information Technology; Medical Informatics Applications; Municipal Management; Local Health Systems 


\section{INTRODUÇÃO}

Na saúde pública brasileira, a gestão do Sistema Único de Saúde (SUS) é compartilhada nas três esferas de governo, em alinhamento com os processos de descentralização e regionalização, que permitiram que cada município, que é conhecedor de suas demandas, possa agir de acordo com as necessidades locais e estabelecer um processo de regulação da atenção à saúde, propiciando mecanismos para o fortalecimento da capacidade de gerir o sistema ${ }^{1}$.

Paralelamente, o avanço da municipalização auferiu aos gestores municipais a responsabilidade de assumir o processo de construção do SUS em seu território, por meio de articulação de planejamento, pactuações para estruturar o acesso e a assistência compatíveis às necessidades locais ${ }^{2,3}$. No entanto, a forma como se processou a municipalização conferiu desigualdades na distribuição de serviços e na fragmentação em ações de projetos e programas 4 .

Para avançar o projeto de universalização da atenção à saúde de forma equitativa, é importante que os gestores das três esferas de governo tenham conhecimento do funcionamento do sistema de saúde e seus arranjos organizacionais ${ }^{5}$. O conhecimento sobre a dinâmica do SUS fortalece as estratégias de planejamento em saúde para superação das lacunas de integração na política pública de saúde.

Para o exercício da gestão em saúde, são necessárias habilidades de planejamento, execução e controle, além de estratégias para sistematizar e formalizar o planejamento em saúde nos níveis de atenção à saúde ${ }^{6}$. Assim, as ações dos gestores em saúde precisam assegurar, por meio do planejamento, a alocação de recursos humanos, materiais e tecnológicos, o monitoramento e a avaliação das ações dos serviços de saúde, além de direcionar a execução de uma complexa rede de assistência; tal demanda implica em cooperação técnica e alinhamento de ações planejadas pelos gestores ${ }^{7}$.

Nessa esfera, priorizar o planejamento como estratégia de gestão acarretará aumento de atividades, controle dos custos, monitoramento e avaliação das ações, por meio da combinação de objetivos pré-determinados ${ }^{8}$. Para auxiliar o processo de planejamento e tomada de decisão, a disponibilidade e o fácil acesso aos dados secundários são mandatórios, contexto em que se inserem os Sistemas de Informação em Saúde (SIS) ${ }^{9}$. A implementação e o uso dos SIS são recomendados por sua capacidade de sensibilizar, fomentar alianças e mobilizar recursos ${ }^{10}$.

Os SIS são considerados indispensáveis para a prática da gestão ${ }^{11}$, pois fortalecem as possibilidades de conhecimento da realidade local e servem de base para a tomada de decisão ${ }^{12}$, além de contribuir para que o gestor alinhe as demandas com as responsabilidades dos serviços de saúde ${ }^{13}$ e reduza as incertas atinentes à gestão em saúde ${ }^{14}$. 
A gama de oportunidades de informações geradas pelos serviços de saúde e armazenadas nos SIS é ampla, mas o seu uso é subutilizado; logo, os dados armazenados precisam ser lapidados e interpretados para suportar o avanço da equipe de gestão na análise e melhoria do processo de tomada de decisão 15,16 .

No âmbito de saúde, algumas empresas utilizam os recursos dos SIS para a elaboração de transações de forma mais eficiente e eficaz, incorporando novas tendências de tecnologias da informação, na perspectiva de proporcionar benefícios diretos e indiretos para a gestão em saúde ${ }^{17}$.

Nesse sentido, considerando as potencialidades relacionadas ao uso de tecnologias da informação no apoio à gestão, a presente investigação teve por objetivo analisar, a partir do suporte de assessoria de informação, as demandas de gestores de saúde para a tomada de decisão.

\section{MÉTODO}

Trata-se de uma pesquisa documental descritiva adotando o método de estudo de caso múltiplo, por permitir compreender o fenômeno em diferentes contextos ${ }^{18}$, bem como o mais utilizado nos estudos com sistemas de informação ${ }^{19}$.

O campo de estudo foi uma empresa com sede no município de Pato BrancoPR, que atua na área desde 2003, e nos últimos anos expandiu sua atuação no segmento por todo território nacional, em Estados como Paraná, Santa Catarina, Rio Grande do Sul, São Paulo, Rio de Janeiro, Sergipe, Bahia, Ceará, entre outros. Para seleção dos munícipios, optou-se por amostragem não aleatória intencional, com seleção de nove dos 250 munícipios atendidos pela empresa. A justificativa da escolha dos municípios pautou-se em razão de atuação consolidada e pioneira nas regiões oeste de Santa Catarina e sudeste do Paraná, maior demanda de chamados nos últimos cinco anos, resultando na seleção dos municípios de Chapecó e Caçador (Santa Catarina), Francisco Beltrão, Pato Branco, Clevelândia, Pinhais, Itapejara D'Oeste, Mariópolis e Palmas (Paraná).

Os dados foram extraídos do sistema de informações da empresa, destinado à assessoria técnica dos clientes, no segundo semestre de 2019. A composição do banco de dados contou com o suporte online do setor de tecnologia da informação da empresa. Foram considerados elegíveis os chamados realizados pelos gestores de saúde no período de junho de 2018 a junho de 2019.

A estratégia utilizada para coleta de dados é apresentada na Figura 1. 

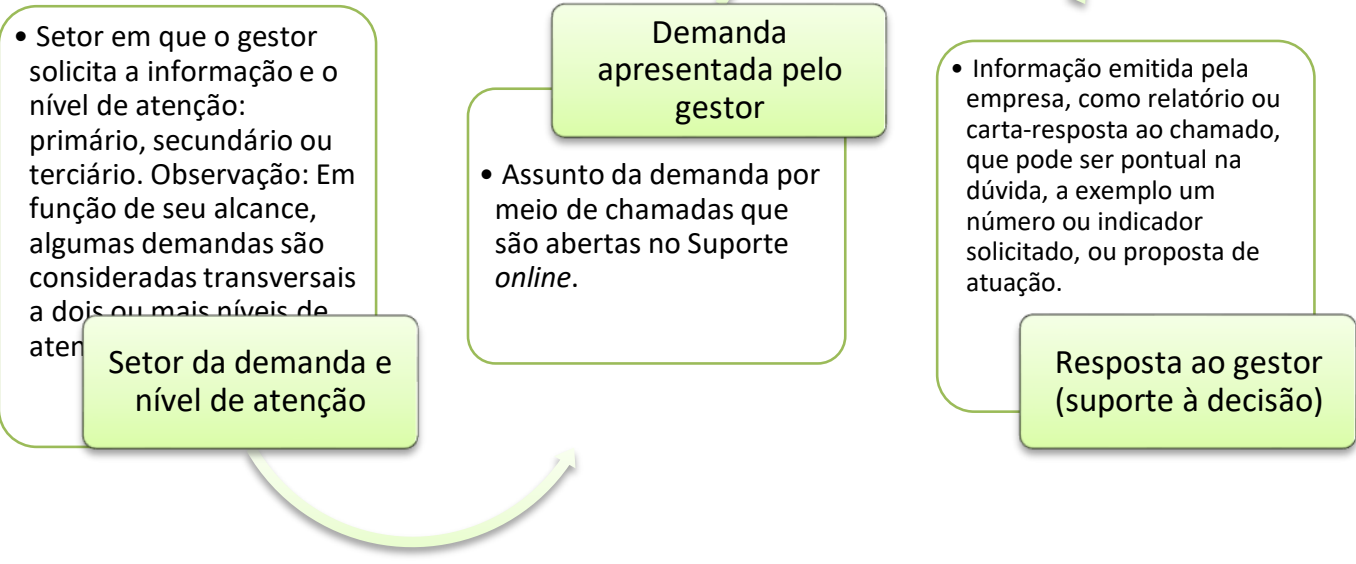

Figura 1. Framework da coleta de dados.

Os dados foram tratados e analisados de modo agregado, de forma a manter o sigilo acerca da demanda de cada município participante. A análise dos dados foi realizada a partir do referencial da análise temática (Braun e Clarke, 2006), com utilização do software Nvivo. O conjunto de respostas emitidas aos gestores são apresentados como produtos para uma possível tomada de decisão, assim essas estratégias significam a possibilidade de melhoria/intervenção a ser implementada pelo gestor a partir das demandas mencionadas nos chamados.

O acesso e utilização dos dados para a finalidade desta investigação foram autorizados pela direção administrativa da empresa, concedida pelo Termo 01/2019, celebrado entre pesquisadores e direção administrativa.

\section{RESULTADOS}

\section{Demandas de gestores para a tomada de decisão}

No período de análise das demandas foram abertos 1.326 chamados. Os chamados foram organizados em assuntos, tendo apresentado maior frequência aqueles relacionados ao fluxo do usuário entre a Estratégia Saúde 
da Família (ESF) e a Unidade de Pronto Atendimento (UPA) $(37,3 \%)$, informações sobre o atendimento na ESF $(16,2 \%)$ e na UPA $(15,0 \%)$, recursos humanos e financeiros $(11,3 \%)$, laboratório $(6,7 \%)$ farmácia e almoxarifado $(5,2 \%)$, Hospital $(3,9 \%)$, ouvidoria $(2,2 \%)$, serviços de odontologia $(2,2 \%)$. As solicitações foram encaminhadas de maneira individual ou em demanda conjunta por um ou mais setores e serviços e serviços de saúde.

O serviço ou setor de origem, bem como o conteúdo da demanda do gestor (Figura 2), revelam que as solicitações dos gestores foram relacionadas, em maior frequência, à assistência ao usuário, tais como informações sobre pacientes, consultas, atendimentos e procedimentos. Para compreensão das ações em saúde, os gestores solicitaram informações sobre motivo do atendimento ou de ausência em consultas, duplicidade de atendimentos, procedimentos e/ou encaminhamento para outros serviços. No âmbito do profissional responsável pelo atendimento ao usuário, foram solicitadas informações de produtividade e conduta de profissionais. Por fim, em menor frequência, foram solicitadas informações sobre o custo do paciente por unidade de atenção em relação ao medicamento, insumos, consultas e exames.

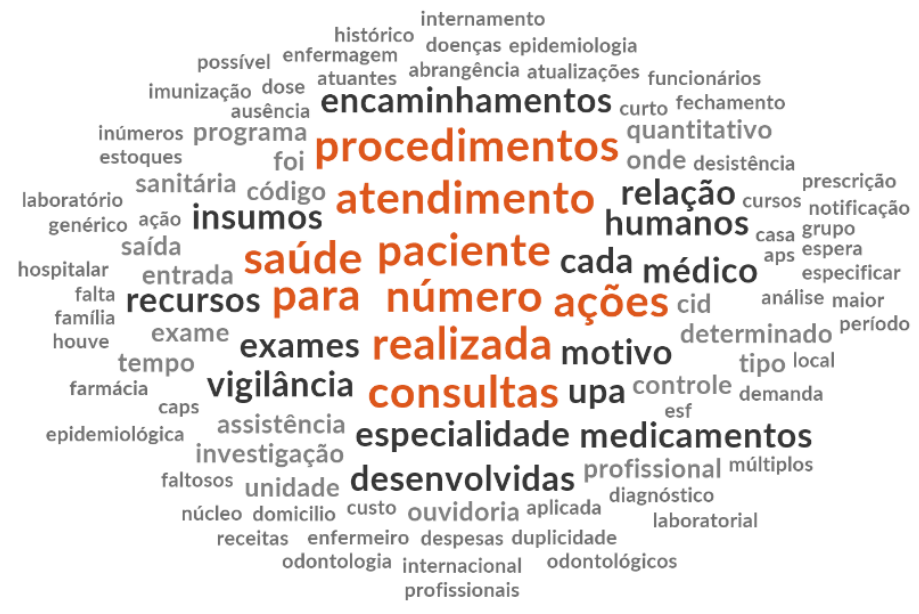

Figura 2. Demandas de chamados abertos por gestores.

Em relação às operações (produção de serviços), os gestores solicitaram informações sobre a quantidade de atendimentos por profissão/especialidade, o tempo gasto por tipo de atendimento e a quantidade de procedimentos odontológicos.

Quanto ao monitoramento do cuidado prestado, foram solicitados relatórios de ações programáticas dos programas de atenção à saúde, quantidade de faltosos em consultas agendadas, desistências de atendimento nas UPAs, quantidade da dispensação de medicamentos nas unidades de saúde, e rastreio de exames realizados por grupos que são acompanhados por 
programas de atenção para verificar se há duplicidade do mesmo exame em um curto período. Além desses, a lista de espera para consultas nas unidades de saúde e a demanda reprimida foi requerida com expressiva frequência.

As coordenações de atenção à saúde, tanto da atenção primária quanto da média e alta complexidade, solicitaram mais relatórios sobre a entrada e saída de insumos, o controle de recursos humanos, tal como o número de profissionais atuantes e especialidades, a quantidade de diárias e transporte fornecido para realização de cursos e atualizações, além do índice de absenteísmo, tanto no trabalho quanto nas dispensas para capacitação.

No setor de vigilância em saúde, a área epidemiológica solicitou relatórios ou panorama de notificação e possíveis investigações realizadas, tendo a área sanitária o levantamento do requerimento de visita, local e conduta realizada, além de compilação das solicitações de atuação a partir de denúncias.

Em resposta aos chamados abertos pelos gestores, a equipe de assessoria forneceu relatórios e dados referentes à produção de atendimentos por diferentes profissionais em determinado setor, categoria profissional, especialidades médicas, programa, procedimento e período. Também foram encaminhados relatórios de reclamações, denúncias, elogios e sugestões; monitoramento de profissionais; tempo médio de atendimento e tempo de espera. No tocante aos recursos financeiros, custos e gastos, foram encaminhados relatórios referentes aos incentivos financeiros, Piso fixo e variável da Atenção Básica (PAB), vencimentos, programas, custo por atendimento/usuário.

\section{Oportunidades proporcionadas no uso de dados para a tomada de decisão}

A partir da análise do material das respostas da empresa de assessoria aos gestores, foi possível identificar possibilidades de suporte que a empresa fornece no uso do sistema para a melhoria da tomada de decisão. O Quadro 1 apresenta uma síntese interpretativa a partir dessa análise.

Quadro 1. Possibilidades de melhoria para a tomada de decisão frente ao suporte da empresa.

- Melhoria no processo de atendimento humanizado por minimizar pacientes em filas.

- Melhor controle do fluxo de pessoas atendidas, de filas de espera, e exames;

- Maior assertividade na tomada de decisões, pois os dados são disponibilizados em tempo real; 
- Segurança dos dados;

- Metas são mais facilmente alcançadas;

- Maior/melhor controle dos dados e recursos;

- Recursos utilizados de maneira mais adequada;

- Maior controle das despesas e das dispensações dos insumos.

\section{Reflexões a partir das demandas dos gestores sob o olhar dos pesquisadores}

$\mathrm{Na}$ análise documental, foi possível identificar dificuldades técnicas dos gestores para extração e análise de dados, uma vez que parcelas das demandas são relativas a atividades como elaboração de gráficos e/ou tabelas para analisar os programas e as ações dos serviços no sistema local de saúde.

As demandas abertas no sistema da empresa e os assuntos que compõem 0 chamado apresentaram oportunidade de identificar necessidades, problemas e dificuldades dos gestores no cotidiano da prática de gestão e, consequentemente, revelar as funções de suporte da empresa aos gestores. Logo, tal análise permite explorar pontos para o desenvolvimento e qualificação da prática de gestão em saúde. O Quadro 2 sintetiza os principais problemas apontados nos chamados dos gestores, que repercutem diretamente na gestão do sistema de saúde.

Quadro 2. Síntese dos principais problemas verificados nos chamados dos gestores identificados em duas dimensões: a gestão e profissional.

\begin{tabular}{|l|l|}
\hline Gestão & $\begin{array}{l}\text { Rotatividade dos funcionários gera problemas na } \\
\text { alimentação do SIS e na produção do serviço; } \\
\text { - Falta de tempo para a capacitação de um novo } \\
\text { funcionário; } \\
\text { - Falta de equipamentos (computadores) suficientes } \\
\text { e/ou dificuldade em relação à disponibilidade do } \\
\text { sinal de internet, principalmente em área rural, ou } \\
\text { instabilidade no sistema, atrasando a resposta ou } \\
\text { interação com o suporte da empresa; }\end{array}$ \\
- Dificuldade em obter indicadores em relação aos \\
atendimentos dos usuários nas unidades de saúde; \\
- Perda de tempo organizando dados e demorava
\end{tabular}




\begin{tabular}{|l|l|}
\hline & \multicolumn{1}{|c|}{$\begin{array}{l}\text { em chegar a um consenso para a tomada de } \\
\text { decisões. }\end{array}$} \\
\hline Profissionais & $\begin{array}{l}\text { Falta de adesão por parte dos funcionários ao } \\
\text { sistema de informação implantado; } \\
\text { - Dificuldade em elaborar e analisar planilhas e } \\
\text { relatórios; } \\
\text { - Pouca responsabilidade na alimentação do sistema } \\
\text { de informação. }\end{array}$ \\
\hline
\end{tabular}

\section{DISCUSSÃO}

Falar em gestão em saúde sem um sistema de suporte de dados é inadmissível. As informações precisas e constantes são subsídios para gerir e compartilhar conhecimento produzido em larga escala nos serviços.

Nesse estudo, verificou-se que gestores têm utilizado o serviço de assessoria técnica como canal para resolver as dificuldades encontradas no dia a dia. Cabe destacar que, o SUS possui o um suporte de sistema de informação para auxiliar o Ministério da Saúde e as vinte e sete secretarias estaduais de saúde, entre outros órgãos em todas as regiões do país, no intuito de fortalecer o SUS a partir de uma ferramenta que promove cooperação técnica em informática para apoiar a gestão no planejamento, na avaliação e na operacionalização de ações e de serviços de saúde 20,21

O setor saúde é complexo, o que amplia a dificuldade de transportar essas informações para os sistemas de informação em saúde, tanto na necessidade de definições de padrões quanto na estratégia de unificar uma linguagem que possa ser compatível a todas as empresas que trabalham com esses dados ${ }^{22}$. Outra questão é a livre iniciativa privada com pouca orientação e regulação sobre as diferentes formas de produzir SIS que acabam fragmentando ainda mais as ações da política de informática em saúde ${ }^{4}$.

No caso estudado, percebe-se a reponsabilidade do gestor em saúde com o nível primário de atenção pela demanda de solicitações referentes às informações sobre a produção nas unidades básicas de saúde, o que reforça a ação da municipalização da atenção. Outro aspecto é a solicitação do fluxo assistencial entre as unidades básicas e a UPA, exibindo que essa maior proporção de demandas assistenciais evidencia necessidade de articular ações entre os serviços de saúde. Assim, a coordenação da rede de atenção torna-se essencial para superar fragilidades de atenção.

A organização do fluxo de atenção a partir da APS é uma recomendação internacional para sistemas de saúde, se fortalece na proposta de melhorar a interação dos serviços que compõe a rede de atenção ${ }^{23}$. No entanto, é a partir 
do conhecimento do perfil epidemiológico da população que se estabelece uma boa coordenação dos fluxos de atenção na rede, exigindo do gestor capacidade de planejamento sobre as prioridades reguladoras da rede de atenção ${ }^{24}$.

Nesse sentido, o gestor em saúde precisa conhecer a importância de identificar os problemas cotidianos que ocorrem nos serviços de saúde sejam com os dados processados ou durante seu processamento para assim enfrenta-los por meio de ferramentas de gestão a exemplo o planejamento ${ }^{25}$. Os relatórios e planilhas oportunizam informações importantes para o planejamento e gerenciamento dos processos de gestão ${ }^{26}$.

A consolidação da organização dos serviços de saúde e da própria rede de atenção à saúde parte de um bom planejamento ${ }^{27}$ que requer do gestor responsabilidade no uso dos Sistemas de Informação em Saúde (SIS) para a melhora da regulação da atenção.

Os SIS tem sido o apoio dos gestores para respostas mais rápidas frente à crescente demanda do uso de serviços de saúde ${ }^{28}$. Considera-se que os dados gerados pelos SIS apoiam a segurança e qualidade da informação e o planejamento a ser repassado aos colaboradores que operam os fluxos no sistema de saúde ${ }^{29}$.

A identificação de uma necessidade a ser enfrentada, propicia um rico material para desenvolver proposta de intervenções em qualquer nível de atenção, ente federativo e órgãos competentes ${ }^{30}$. No entanto, é necessário responsabilidade com um processo avaliativo adequado que mobilize recursos necessários capazes de promover ações para solucionar os problemas encontrados ${ }^{31}$.

Por isso, destaca-se que é pertinente recomendar e incentivar a alimentação dos SIS, que devem ser constantes e rápidas para propiciar condições de previsão de possíveis agravamentos da situação de saúde. Os dados devem ser registrados assim que o evento ocorre e sua precisão servirá como característica essencial na agilidade para embasar a tomada de decisão ${ }^{32}$.

Enfatiza-se que alimentar os SIS do SUS persiste como exigência para repasse de recursos para implantação e custeio, bem como influenciará na atuação dos gestores ${ }^{33}$. Múltiplos atendimentos de um mesmo usuário em locais diferentes em dias próximos e com as mesmas queixas geram custo elevado ao sistema de saúde. Assim, recomenda-se integração dos sistemas de atendimento e informação para que permita visualização dos usuários atendidos, suas queixas e insumos dispensados, pois a implantação de software que gerencie de forma integrada o prontuário do paciente propicia um recurso que fortalece a gestão assistencial ${ }^{34}$.

Conforme foi exposto, fomentar o uso dos SIS é propiciar à gestão melhores estratégias para garantir a atenção à saúde, gerar benefícios na organização do sistema de saúde e reduzir os custos e do tempo de análise da situação de saúde ${ }^{35}$. Nesse sentido, analisar os indicadores de saúde associados aos sistemas de informações possibilita avançar nos processos de planejamento, monitoramento e avaliação dos serviços de saúde ${ }^{36}$. 
Ressalta-se que, a função dos SIS é oportunizar a construção de indicadores de saúde com relevância para análise da situação de saúde, espaço que compete à gestão enfoque para a tomada decisão ${ }^{37}$. Dados e indicadores são fundamentais para o monitoramento, sem essa informação os processos não são avaliados e fragiliza-se o alcance das metas estabelecidas para melhorar 0 desempenho do sistema de saúde.

O uso do sistema de informação oportuniza agilidade no processo de tomada de decisão. Contudo, é necessário que também haja investimento na avaliação dos SIS. Precisamente, é importante ter um modelo claro de forma conceitual e prática, que explicite o que é fundamental no banco de dados para a construção de indicadores bem como uma política eficaz de um sistema de gestão que se baseia nas informações produzidas por seus colaboradores para facilitar a tomada de decisão ${ }^{38}$.

A forma como os dados são disponibilizados permitirá ações concisas para cada necessidade. Destarte, o acesso a esses dados e a essas informações possibilitam ao gestor uma análise descentralizada, o que também pode ser de forma desagregada quando se tem interesse em um ambiente específico, servindo como instrumento de apoio a tomadas de decisões.

Cabe ressaltar que, a partir do dinamismo dos processos que envolvem cada situação ou setor dentro de uma secretaria municipal de saúde, a incorporação de mudanças vividas no espaço político serve como articulador entre equipes de saúde e gestão, na definição de prioridades para o planejamento das ações. Portanto, a comunicação entre as equipes de gestão e da assistência são mandatórias para utilização de indicadores fidedignos no monitoramento das ações e, consequentemente, a mudança de novas definições para suprir o desafio de gerir os SIS ${ }^{39}$.

O futuro das redes e tecnologias sem fio cada vez é mais promissor o que viabiliza redes digitais de área local e de longa distância com alto desempenho, essa tendência de mobilidade oferece conveniências como computadores portáteis (palmtops) e agendas eletrônicas computadorizadas (PDAs) assim esses equipamentos têm a vantagem de ter a portabilidade e conectividade sem fio, bem como sua aplicação na telemedicina ${ }^{40,41}$.

Definir estratégias na gestão junto com a Tecnologia de informação (TI) demandará recursos e exigirá clareza do tipo de informação, o fluxo e os acessos em diferentes níveis de forma confiável, evitando erros, desperdícios, abusos e fraudes; então, os sistemas devem conter informações de informações confiáveis e integradas junto aos usurários do sistema e gestores 42.

Dentre as funções dos sistemas de informação, está a construção de indicadores de saúde com relevância para a gestão, com foco nas decisões a serem tomada com a incorporação de uma prática sistemática para melhorar diversos níveis da atenção em saúde para uma reorganização dos serviços prestados ao coletivo ou a comunidade ${ }^{43}$. 
Para cada realidade é preciso examinar os indicadores a fim de atender às necessidades daquele serviço. Nesta lógica, a utilização das informações dos SIS deve superar a função técnico-burocrático, representada pelo esforço em alimentar os dados solicitados, para contribuírem de forma relevante nos processos de planejamento e monitoramento das ações dos serviços de saúde 44 .

Assim, o uso dos SIS como ferramenta de análise do processo de trabalho é pertinente tanto para o gestor quanto para o profissional, independentemente do nível de atenção, pois amplia a visão dos problemas e permite melhorias na condução da prática ${ }^{45}$.

Notoriamente, é percebido que a tomada de decisão em saúde pública é desafiadora e rodeada de incertezas, mas este processo é extremamente necessário ${ }^{31}$. Na utilização de um SIS para a tomada de decisão, as demandas e as necessidades dos gestores podem ser influenciadas por fatores como cultura organizacional, políticas públicas, recursos humanos sem a necessária capacitação, desenvolvimento de atividades burocratizadas e estrutura física incipiente para o uso de tecnologias informacionais, além da falta de integração dos sistemas que não favorecem a análise mais ampliada da situação apresentada ${ }^{46}$.

Tem-se verificado a utilização limitada dos SIS para a tomada de decisão por parte dos gestores, o que expressa dificuldade em direcionar as ações e as intervenções em saúde, comprometendo o planejamento, que deveria minimizar os problemas cotidianos das necessidades dos usuários ao acessar o sistema de saúde ${ }^{47}$. Gestores devem perceber que o SIS é uma ferramenta extremamente útil para ser levada em consideração no momento da tomada de decisão, sua pouca utilização na gestão dos serviços em saúde sinaliza dificuldades de desempenhar funções de gestão ${ }^{48}$.

Entende-se que a utilização dos SIS na gestão, tanto por uma questão burocrática quanto pela oportunidade do uso desses dados para superar problemas recorrentes dos serviços, tem esbarrado na capacidade do gestor compreender o uso do sistema como prática de gestão ${ }^{49}$. Para que o SIS possa ser fortalecido, além de propiciar melhoria na rede de tecnologia e na forma como se processa os dados, é necessário que o gestor possua conhecimento dos serviços e das fontes de informação em saúde, conhecimentos das políticas de saúde vigentes (nacional e internacional), problemas e tendências que impactam as tecnologias em saúde; bem como habilidades para adquirir informação registrada em qualquer suporte tanto em sua forma original como em produtos elaborados a partir dela ${ }^{13}$.

As limitações desse estudo abordam a dimensão do uso somente dos chamados aberto por gestores, o que especificamente direciona para eventuais problemas da gestão sem ter oportunizado a compreensão a partir da fala dos gestores, recomendando-se assim ampliar a análise nessa perspectiva. Os resultados apresentados nesse estudo possibilitam explorar a elaboração de pesquisa que compreenda o processo de trabalho da gestão no uso cotidiano dos SIS. 


\section{CONSIDERAÇÕES FINAIS}

Em suma, as maiores demandas pelos gestores predominam por verificação da relação entre o tempo da consulta e a carga horária de cada médico; diagnosticar qual é o tempo de espera em filas em cada unidade; controlar os fluxos de recebimento e entrega dos medicamentos; controlar o encaminhamento de solicitações de exames e também as suas retiradas por unidade com o objetivo de diminuir o custo laboratorial; controlar o número de consultas que são feitas somente para retirada de receitas de medicamentos; diagnosticar o número de cidadãos que consultam pela Operadora de Saúde, mas procuram a Unidade para fazer os exames de forma gratuita; critérios de encaminhamentos para a UPA, verificação da atuação das equipes da ESF.

O reconhecimento a partir de uma análise das demandas e necessidades de gestores municipais para a tomada de decisão nesse grupo específico auxiliou a empresa a reconhecer as possibilidades de ampliar o apoio reconhecendo também oportunidade de desenvolver capacitação abordando as demandas e potencializando a função empresarial no assessoramento à gestão. Nesse sentido, destaca-se que pactuar o envolvimento das pessoas, bem como 0 monitoramento e a avaliação no cotidiano dos gestores impactam positivamente na condução de uma tomada de decisão.

A assertividade da tomada de decisão depende de infraestrutura tecnológica, capacidade de análise e avaliação dos SIS para formular a informação correta. Desta forma, a capacitação de recursos humanos capazes de analisar o processo de implantação de uma política pública é imprescindível para a gestão do SUS.

Por fim, acredita-se que a reorganização do processo de trabalho, norteada pela adequada utilização de informações de saúde e sistemas de informação, alcançará a resolução de problemas recorrentes e auxiliará na tomada de decisão oportuna por parte dos gestores em saúde.

\section{REFERÊNCIAS}

1. Viana AL d'Ávila, Bousquat A, Melo GA, Negri Filho A, Medina MG. Regionalization and Health Networks. Cien Saude Colet [Internet]. 2018 Jun;23(6):1791-8. Available from: http://dx.doi.org/10.1590/1413$\underline{81232018236.05502018}$

2. Sauter AMW, Girardon-Perlini NMO, Kopf ÁW. Politics of health's regionalization: from the transaction rules to the Pact for Health. Rev Min Enferm [Internet]. 2012 [cited 2020 Oct 10];265-74. Available from: http://www.reme.org.br/artigo/detalhes/528

3. Coriolano MWL, Albuquerque GA, Araújo NS, Oliveira MA, Lima MM. Living the process of the municipalization of SUS in Juazeiro do Norte, Ceará 
State. Cien Saude Colet [Internet]. 2010 Aug;15(5):2447-54. Available from: https://doi.org/10.1590/S1413-81232010000500019

4. Machado JC, Cotta RMM, Soares JB. Reflexões sobre o processo de municipalização das políticas de saúde: a questão da descontinuidade político-administrativa. Interface - Comun Saúde, Educ [Internet]. 2015 Mar;19(52):159-70. Available from:

http://www.scielo.br/scielo.php?script=sci arttext\&pid=S1414$\underline{32832015000100159 \& \operatorname{lng}=\text { pt\&tlng }=\text { pt }}$

5. Paim JS. Thirty years of the Unified Health System (SUS). Cien Saude Colet [Internet]. 2018 Jun;23(6):1723-8. Available from:

https://doi.org/10.1590/1413-81232018236.09172018

6. Fernandes BHR, Berton LH. Administração Estratégica. 2nd ed. São Paulo: Saraiva; 2012. 272 p.

7. Mascarenhas GC, Shimizu HE. Os desafios do planejamento regional compartilhado à luz do Decreto $\mathrm{n}^{\circ} 7508$ : um estudo de caso Sobral - Ceará. In: Silva RM da, Jorge MSB, Silva Júnior AG, editors. Planejamento, gestão e avaliação nas práticas de saúde [Internet]. 1st ed. Fortaleza: EdUECE; 2015. p. 72-93. Available from: http://www.uece.br/ppsacwp/wpcontent/uploads/sites/37/2016/04/PLANEJAMENTO-GESTAO-EAVALIACAO-NAS-PRATICAS-DE-SAUDE-EBOOK-548pg.pdf

8. Rivera FJU. Planejamento de Saúde [Internet]. 2009 [cited 2020 Oct 10]. Available from:

http://www.sites.epsjv.fiocruz.br/dicionario/verbetes/plasau.html

9. Organização Mundial da Saúde. Diretrizes da OMS sobre política de saúde e apoio sistémico para a otimização de programas de agentes comunitários de saúde [Internet]. Genebra; 2019. Available from:

https://apps.who.int/iris/bitstream/handle/10665/275474/9789248550362por.pdf?ua $=1$

10. Organização Pan-Americana da Saúde. Plano Estratégico da Organização Pan-Americana da Saúde 2014-2019 [Internet]. Washington, D.C.; 2014. Available from: https://iris. paho.org/bitstream/handle/10665.2/7654/CD53OD345-p.pdf?sequence $=15$ \&isAllowed $=y$

11. Brandão ACS, Silva JRA. A contribuição dos sistemas de informação em saúde (SIS) para o processo de auditoria do SUS. Rev Atualiza Saúde [Internet]. 2015 [cited 2020 Oct 10];1(1):17-24. Available from: http://atualizarevista.com.br/article/v1-n1-a-contribuicao-dos-sistemas-deinformacao-em-saude-sis-para-o-processo-de-auditoria-do-sus/

12. Casa Civil, Ministério da Transparência, Controladoria-Geral da União, Ministério da Fazenda, Ministério do Planejamento Desenvolvimento e Gestão, Aplicada I de PE. Avaliação de políticas públicas: guia prático de análise ex post. v. 2. Brasília: Casa Civil da Presidência da República; 2018. $301 \mathrm{p}$. 
13. Fernandes VA, Hommerding NMS. Gestor da informação em saúde: promotor do conhecimento na tomada de decisão. Rev Bras Bibliotecon e Doc [Internet]. 2019 [cited 2020 Oct 10];15(1):111-32. Available from: https://febab.emnuvens.com.br/rbbd/article/view/1141

14. Garcia PT, Reis RS. Gestão pública em saúde: sistemas de informação de apoio à gestão em saúde [Internet]. 1st ed. São Luis: EDUFMA; 2016. 53 p. Available from: https://ares.unasus.gov.br/acervo/html/ARES/7370/1/GP2U3.pdf

15. Santos TO, Pereira LP, Silveira DT. Implantação de sistemas informatizados na saúde: uma revisão sistemática. Rev Eletrônica Comun Informação e Inovação em Saúde [Internet]. 2017 Sep 29;11(3). Available from: https://doi.org/10.29397/reciis.v11i3.1064

16. Gleriano JS, Fabro GCR, Tomaz WB, Goulart BF, Chaves LDP. Reflections on the management of Brazilian Unified Health System for the coordination in facing COVID-19. Esc Anna Nery [Internet]. 2020;24(spe). Available from: http://dx.doi.org/10.1590/2177-9465-ean-2020-0188

17. Contreras Pinochet LH, Lopes AS, Silva JS. Inovações e Tendências Aplicadas nas Tecnologias de Informação e Comunicação na Gestão da Saúde. Rev Gestão em Sist Saúde [Internet]. 2014 Dec 1;03(02):11-29. Available from: https://doi.org/10.5585/rgss.v3i2.88

18. Benbasat I, Goldstein DK, Mead M. The Case Research Strategy in Studies of Information Systems. MIS Q [Internet]. 1987 Sep;11(3):369. Available from: https://www.jstor.org/stable/248684?origin=crossref

19. Oliveira M, Maçada ACG, Goldoni V. Strength and weaknesses of the case study method when applied to area of information systems. REGE Rev Gestão [Internet]. 2009 Mar 1 [cited 2020 Dec 4];16(1):33-49. Available from: https://www.revistas.usp.br/rege/article/view/36660

20. Cunha EM da, Vargens JM da C. Sistemas de informação do Sistema Único de Saúde. In: GONDIM GM de M, CHRISTÓFARO MAC, MIYASHIRO GM, editors. Técnico de vigilância em saúde: fundamentos [Internet]. 1st ed. Rio de Janeiro: EPSJV; 2017. p. 71-112. Available from: https://www.arca.fiocruz.br/bitstream/icict/39907/2/Técnico de Vigilância em Saúde v.2 - Sistemas de informação do Sistema Único de Saúde.pdf

21. Lima AC, Januário MC, Lima PT, Silva WM. DATASUS: O USO DOS SISTEMAS DE INFORMAÇÃO NA SAÚDE PÚBLICA. Rev da FATEC Zo Sul - Análise e Desenvolv Sist [Internet]. 2015;1(3):16-31. Available from: http://revistarefas.com.br/index.php/RevFATECZS/article/view/27

22. Moreno RA. Interoperabilidade de Sistemas de Informação em Saúde. J Heal Inf [Internet]. 2016 [cited $2021 \mathrm{Feb} 25$ ];8(3). Available from: http://www.hipaajournal.com/major-2016-healthcare-data-breaches-midyear-summary-3499/ 
23. Nascimento LC do, Viegas SM da F, Menezes C, Roquini GR, Santos TR. O SUS na vida dos brasileiros: assistência, acessibilidade e equidade no cotidiano de usuários da Atenção Primária à Saúde. Physis Rev Saúde Coletiva [Internet]. 2020;30(3). Available from:

http://www.scielo.br/scielo.php?script=sci arttext\&pid=S010373312020000300612\&tlng=pt

24. Bernardino Junior SV, Medeiros CRG, Souza CF de, Kich J, Alves AM, Castro LC de. Processos de encaminhamento a serviços especializados em cardiologia e endocrinologia pela Atenção Primária à Saúde. Saúde em Debate [Internet]. 2020 Sep;44(126):694-707. Available from: http://www.scielo.br/scielo.php?script=sci arttext\&pid=S0103$11042020000300694 \& \operatorname{lng}=\mathrm{pt}$

25. Martins CC, Waclawovsky AJ. Problemas e Desafios Enfrentados pelos Gestores Públicos no Processo de Gestão em Saúde. Rev Gestão em Sist Saúde [Internet]. 2015 Jun 1;04(01):100-9. Available from: http://dx.doi.org/10.5585/rgss.v4i1.157

26. Galdino SV, Reis ÉMB, Santos CB, Soares FP, Lima FS, Caldas JG, et al. Ferramentas de qualidade na gestão dos serviços de saúde: revisão integrativa de literatura. Rev Eletronica Gestão Saúde [Internet]. 2016 Jul 29 [cited 2020 Oct 10];0(supl.):1023. Available from:

https://periodicos.unb.br/index.php/rgs/article/view/3569

27. Ferreira J, Celuppi IC, Baseggio L, Geremia DS, Madureira VSF, Souza JB. Regional planning of health services: what do the managers say? Saúde e Soc [Internet]. 2018 Jan;27(1):69-79. Available from: https://doi.org/10.1590/s0104-12902018170296

28. Trottmann P, Corrêa V. Planejamento estratégico orientado ao setor público. Editora Senac São Paulo; 2019. 256 p.

29. Jannuzzi CASC, Falsarella OM, Sugahara CR. Information system: a conceptual understanding for application in business organisations. Perspect em Ciência da Informação [Internet]. 2014 Dec;19(4):94-117. Available from: https://doi.org/10.1590/1981-5344/1927

30. Louvison MCP. Regionalization of health systems as a response to territorial inequalities: a necessary debate. Cad Saude Publica [Internet]. 2019;35(suppl 2). Available from: https://doi.org/10.1590/0102$\underline{311 \times 00116019}$

31. Tanaka OY, Tamaki EM. The role of evaluation in decision-making in the management of health services. Cien Saude Colet [Internet]. 2012 Apr;17(4):821-8. Available from: https://doi.org/10.1590/S141381232012000400002

32. Sharda R, Delen D, Turban E. Business Intelligence e Análise de Dados para Gestão do Negócio. 4th ed. Bookman; 2019. 614 p. 
33. Jaccoud L, Vieira FS. Autonomia, integralidade e desafios de coordenação no SUS. In: Jaccoud L, editor. Coordenação e relações intergovernamentais nas políticas sociais brasileiras [Internet]. Brasília: IPEA; 2020. p. 53-80. Available from: http://dx.doi.org/10.38116/978-65-5635-005-9/cap2

34. Souza MC, Tomazelli R, Vasconcelos CRM. Prontuário Eletrônico: um Determinante no Gerenciamento de Cliente/Paciente em um Sistema de Informação Hospitalar. Rev Espac [Internet]. 2016 May 29 [cited 2020 Oct 10];37(4). Available from:

https://www.revistaespacios.com/a16v37n14/16371423.html

35. Brito JF, Silva RC da, Santos BRP, Mello MRG de, Martínez-Ávila D. Arquitetura da informação no contexto da informação em saúde: um olhar para o website do COVID-19 no Brasil. AtoZ novas práticas em informação e conhecimento [Internet]. 2020 Dec 8;9(2):183. Available from: https://revistas.ufpr.br/atoz/article/view/75091

36. Albuquerque C, Martins M. Performance indicators in the Unified Health System: an assessment of advances and gaps. Saúde em Debate [Internet]. 2017 Mar;41(spe):118-37. Available from: https://doi.org/10.1590/0103$\underline{11042017 s 10}$

37. Weigelt LD, Mancio JG, Petry EL da S. Health indicators in the view of managers of the municipalities in the 13 th regional health district - RS. Barbar [Internet]. 2012 [cited 2020 Oct 10];(36):191-205. Available from: http://pepsic.bvsalud.org/scielo.php?script=sci abstract\&pid=S010465782012000100012\&lng=pt\&nrm=iso\&tlng=en

38. Carnut L, Narvai PC. Performance evaluation of health systems and management in the Brazilian public administration. Saúde e Soc [Internet]. 2016 Jun;25(2):290-305. Available from: https://doi.org/10.1590/S0104$\underline{12902016144614}$

39. Grimm SCA, Tanaka OY. Municipal Monitoring Panel: Bases for building a health service management tool. Epidemiol e Serviços Saúde [Internet]. 2016 Sep;25(3):585-94. Available from: http://dx.doi.org/10.5123/S1679$\underline{49742016000300014}$

40. Contreras Pinochet LH. Trends of Information Technology in Health Management . Mundo saúde [Internet]. 2011 [cited 2020 Oct 10];35(4):38294. Available from: http://bvsms.saude.gov.br/bvs/artigos/tendencias tecnologia informacao g estao saude.pdf

41. Santos A, Firmino R, Soto J, Medeiros D, Mattos D, Albuquerque C, et al. Aplicações em redes de sensores na área da saúde e gerenciamento de dados médicos: tecnologias em ascensão. In: Minicursos SBCAS 2020 [Internet]. SBC; 2020. p. 137-86. Available from: https://sol.sbc.org.br/livros/index.php/sbc/catalog/view/47/213/434-1 
42. Bittar OJN, Biczyk M, Serinolli MI, Novaretti MCZ, Moura MMN. Health information systems and their complexity. Rev Adm em Saúde [Internet]. 2018 Jan 12;18(70). Available from: http://dx.doi.org/10.23973/ras.70.77

43. Santos BRP, Damian IPM. Análise da Gestão da Informação na Atenção Básica em Saúde: um estudo em Unidades de Saúde da Família. Informação Soc Estud [Internet]. 2019 Sep 30 [cited 2020 Oct 10];29(3):171-94. Available from:

https://periodicos.ufpb.br/ojs2/index.php/ies/article/view/45547

44. Pereira MM, Maciel ALS, Melo PR, Fontenele S de B. Sistema de informação geográfica como ferramenta de subsídio a gestão pública no Brasil [Internet]. 2020 [cited 2020 Oct 10]. (Sistema de informação geográfica como ferramenta de subsídio a gestão pública no Brasil). Available from: https://proceedings.science/sbsr-2019/papers/sistema-deinformacao-geografica-como-ferramenta-de-subsidio-a-gestao-publica-nobrasil\#

45. Cavalcante Junior FC, Inocêncio Junior J, Silva PPC, Ceolin AC. Sistemas de Informação Gerenciais na visão e atuação do Controlador geral em um município pernambucano. Refas - Rev Fatec Zo Sul [Internet]. 2019 Nov 22 [cited 2020 Oct 10];6(2):18-32. Available from: http://www.revistarefas.com.br/index.php/RevFATECZS/article/view/358

46. Quites HF de O. Barreiras do uso da Informação em Saúde na tomada de decisão municipal: uma Revisão de Literatura. Rev Eletronica Gestão Saúde [Internet]. 2016 Jul 29 [cited 2020 Oct 10];0(supl.):1011. Available from: https://periodicos.unb.br/index.php/rgs/article/view/3567

47. Lima KWS, Antunes JLF, Silva ZP. Perception of managers on the use of indicators in health services. Saúde e Soc [Internet]. 2015 Mar;24(1):61-71. Available from: https://doi.org/10.1590/S0104-12902015000100005

48. Pinheiro ALS, Andrade KTS, Silva D de O, Zacharias FCM, Gomide MFS, Pinto IC. HEALTH MANAGEMENT: THE USE OF INFORMATION SYSTEMS AND KNOWLEDGE SHARING FOR THE DECISION-MAKING PROCESS. Texto Context - Enferm [Internet]. 2016;25(3). Available from: http://www.scielo.br/scielo.php?script=sci arttext\&pid=S0104$\underline{07072016000300305 \& \operatorname{lng}=e n \& t \operatorname{lng}=e n}$

49. Vidor AC, Fisher PD, Bordin R. Utilização dos sistemas de informação em saúde em municípios gaúchos de pequeno porte. Rev Saude Publica [Internet]. 2011 Feb;45(1):24-30. Available from: http://www.scielo.br/scielo.php?script=sci arttext\&pid=S0034$89102011000100003 \& \operatorname{lng}=p t \&$ tIng $=p t$ 
Recebido: 27 de março de 2021. Aceito: 03 de abril de 2021

Correspondência: Bruna Moreno Dias. E-mail: bruna.dias@usp.br

Conflito de Interesses: os autores declararam não haver conflito de interesses.

(C) This is an Open Access article distributed under the terms of the Creative Commons Attribution License, which permits unrestricted use, distribution, and reproduction in any medium, provided the original work is properly cited 